Article

\title{
Foetal Music Perception: A Comparison Study between Heart Rate and Motor Responses Assessed by APIB Scale in Ultrasound Exam
}

\author{
Rosana Maria Tristao ${ }^{1,2, *}$, Jose Alfredo Lacerda de Jesus ${ }^{1,2}$, Mônica de Lima Lemos ${ }^{1}$ and Ricardo \\ Dourado Freire $^{3}$ \\ ${ }^{1}$ Neonatal and Maternity Unit of the University Hospital de Brasília, University of Brasília, Campus Darcy \\ Ribeiro, Brasília, 70910900, Brazil; limalemos@yahoo.com.br \\ 2Faculty of Medicine, University of Brasília, Campus Darcy Ribeiro, Brasília, 70910900, Brazil; alfredo@unb.br \\ ${ }^{3}$ Institute of Arts, University of Brasília, Campus Darcy Ribeiro, Brasília, 70910900, Brazil; freireri@unb.br \\ * Correspondence: RM Tristão rmtt@unb.br; Tel.: +55-61-99968-9359
}

\begin{abstract}
Music perception in foetuses has been explored under different theoretical paradigms such as habituation, categorical perception, sound preferences and recall. This study investigated the temporal dimension of music perception through the habituation and sensitization paradigm. Foetuses of 41 pregnant women, mean gestational age of 34.7 weeks $( \pm 2.4)$, were observed during ultrasound exams. Foetuses' reaction to two different tempos (Allegro vs Adagio) and sources (internal vs external) of music stimuli was registered by heart rate variability (HR) and motor response according to the Assessment of Preterm Infants Behaviour scale (APIB) by its factors of movement (MOV) and organization (ORG). A folkloric lullaby, sung and played live with a stringed instrument by a musician, was presented in three stages that were compared to baseline: 1) slow tempo (Adagio), 2) fast tempo (Allegro); and 3) now sung by mother at slow tempo (Adagio). Exploratory analyses showed that all factors increased from baseline to first stage. HR and ORG varied significantly among stages, with HR being the strongest factor. MOV merely detected change from baseline to first stage. ORG decreased for Allegro but increased for maternal Adagio, while HR decreased to near baseline values. ANOVA-repeated measures with gestational age as covariate showed that all measures were sensitive to first music presentation (Adagio), although only HR and ORG differed among stages. Considering estimated marginal means, adjusted for gestational age, HR presented a sensitization pattern throughout stages, but ORG kept habituating to external source and increased to maternal Adagio, suggesting foetal discrimination by sound source. We conclude that foetuses showed different behavioural and physiological responses to external versus internal sound source and musical tempo. The combined use of a behavioural scale (APIB) and HR in foetuses proved to be a valid multidimensional instrument.

Keywords: foetus; music perception; tempo; heart rate variability; ultrasound exam; APIB scale; habituation; sensitization.
\end{abstract}

\section{Introduction}

Fascination with the study of perceptual abilities in the foetus started from the late 1800s, but it was only in 1885 that Preyer carried out the first sensory experiments that detected the hearing ability of foetuses [1]. However, it was in 1980 that investigations began to be systematically controlled, due to the sophisticated technology of ultrasound devices and the possibility of monitoring foetal physiology. The main methods for detection of foetal responses to stimuli that induced behavioral and physiological changes, particularly in heart rate variability (HR) and body movements, have been based on devices such as electrocardiographs (golden standard), and on electrocardiographic or magnetocardiographic recordings [1-3].

The hearing process begins with the differentiation of sensory cells, and cochlear maturity takes place at about 20 gestational weeks. The second phase of this process refers to the development of the auditory cortex axis from cochlea to brainstem, in the third trimester, with its myelination reaching adult-like functioning at the end of the first year of life outside the womb. Hence, there is 
evidence that foetuses can hear from at least the last trimester of pregnancy [4,5]. Within thirty weeks of gestation, the foetus has the capacity to respond to white noise of $110 \mathrm{db}$ SPL, with variation in HR and body movement, and it is estimated that the attenuation caused by the womb environment is around $35 \mathrm{~dB}$ [6]. Foetuses between 26-34 weeks of gestation are able to discriminate vowel sounds between 100 and $110 \mathrm{~dB}$ [7] and between 36-40 gestational weeks respond to speech stimuli of 83 to $95 \mathrm{~dB}$ SPL, displaying HR decelerations for vowel sounds [8]. Near-term foetuses could also discriminate the reversal of pairs of consonants to vowel sounds, as well as a change in gender of the speaker $[9,10]$. The findings showed that the closer the end of pregnancy, the greater the magnitude and frequency of response and the lower the latency [1, 11, 12].

The hypothesis that foetuses recognize and store human voices and language to which they are exposed during pregnancy is supported by studies on babies' preferences. Newborns prefer their mother's voice instead of that of a strange woman, a story told by the mother during late pregnancy compared to a new story, and their native language compared to a foreign language [13-15]. Hence, as the foetus can hear at about 30 weeks, music played in the external environment can be detected in the intra-uterine environment [6]. Some studies have examined the effects of music on foetal behavior using long periods of stimulation. For instance, in a study, 11 pregnant women at 28 weeks' gestation were exposed to 10 minutes of their favourite recorded songs listened to at ear level with an average intensity of $75 \mathrm{~dB}$ (range $65 \mathrm{~dB}$ to $100 \mathrm{~dB}$ ). Increased foetal HR of about five beats per minute was seen to occur 90 seconds after the beginning of the song. There were no changes in foetal body movements and no change in maternal HR. The foetal HR returned to baseline levels within two minutes of the beginning of the song. Since the beginning of the response was late and there was no change in activity, the authors speculated that the foetal response was mediated through the mother's emotional reaction [16]. Similarly, Zimmer et cols postulated that changes in foetus behavior in pregnant women between 34 and 40 weeks of pregnancy are mediated by hormonal changes. In the same way, when exposed to music through headphones on the mother, thus masking the sound stimulus to the foetus, it was observed that foetuses showed a decrease in respiratory activity and increased body movement, as the mother listened to her favourite type of music, classical or rock [7]. Moreover, listening to classical music was linked to a lower number of uterine contractions and to stimulating foetal movement in cardiotocographic parameters [17]. In short, there is evidence that foetuses are able to respond to sound stimuli in the intra-uterine environment, and heartbeat is one of the most consistent measurements used in studies, although data interpretation is hazardous, due to the interplay of confounding variables.

Doppler ultrasound has been used for the study of sensory and perceptual behavior of foetuses, and HR variation has been shown to be more consistent with the observation of the overall foetal movement $[18,19]$. Nevertheless, this area has experienced slow progress in the development of technologies to objectively evaluate foetal movement behavior for clinical application $[2,18]$. There are even authors who discuss how dangerous an ultrasound test may be to foetuses, as some observations of foetal locomotor activity during the ultrasound diagnostic procedure have suggested that foetuses respond unfavourably to modulated ultra-sonic irradiation, and this impact would thus be better explored in simulated wombs [20]. A more frequent line of research that comes out in a literature search is the study of the impact of music therapy on pregnant women and their foetuses or ill neonates [21, 22]. For instance, a study using 3D/4D ultrasound observed the behaviours of elicited mouthing and tongue expulsion in $87 \%$ of 106 foetuses between 14 and 39 gestational weeks for intravaginally emitted music, but not for abdominal emission [23]. However, the measurement of gross motor responses in intra-uterus foetuses remains poorly defined, and reports evaluating gross motor activity through the application of behavioral scales to study foetal response to musical stimuli were not found in the literature [19].

Among the scales that would allow the study of motor behaviour in foetuses or premature newborns there is the Assessment of Preterm Infants Behavior, the APIB [24, 25]. The APIB, based on the Brazelton Newborn Behavioral Assessment Scale (NBAS), provides further refinement of the identification of infants' self-regulatory efforts and thresholds for disorganization, as viewed through aspects of infant behaviours referred to as subsystem interactions [26, 27]. The subsystems of the 
infant are formally observed and considered in terms of their organization, and the items include autonomic, motor, state, attention, and self-regulation factors. In newborns, the exam proceeds through a series of manoeuvres that increase in vigour as well as tactile and vestibular demand. Further, the assessment examines the integrity of the infant's sleep organization, systematically elicited movement repertoire, and availability and quality of social interaction. The stability and organization of the infant's subsystems are continuously evaluated in their mutual interplay with each other and, in turn, in their interplay with the examination's graded demands. For instance, the APIB scale was used in a study in a neonatal unit to investigate the discomfort caused by environmental noise in low-weight neonates in incubators. The infants presented significant physiological alterations in response to environmental noise, registered by HR and by the APIB adaptive behaviour dimension, which varied from 2 (very good) to 6 (moderate to poor) [28].

Hence, this study aimed to observe foetal response to a musical stimulus, which changes in tempo and source location, including both an external singer and the mother, during a prenatal clinical follow-up ultrasound exam. This study took advantage of an ongoing hospital project executed by the authors of this paper, involving music for pregnant women, and it registered the interaction of mother and foetus when listening to music. In this project, the musical stimuli were played live, and the pace of the music was enhanced gradually, taking care to provide a welcoming and no-stress environment for mother-foetus comfort. Thus, it adopted an ecological approach, where the clinical context defined how variables were managed and limited manipulations, such as order of presentation or different sources of stimuli. The foetus' responses were measured by HR variability and APIB scale for the foetus' global movements and attentional organization. The hypothesis of this study is that if foetuses perceive the changing tempo and source of the musical stimuli, they will be able to respond in terms of increased or decreased HR and changes in motor activity, in relation to the sound source.

\section{Materials and Methods}

\subsection{Participants}

This is an observational cross-sectional study in a convenience sample of a tertiary hospital service. The sample consisted of 41 adult pregnant women, carrying singleton foetuses, who attended the Obstetrics and Gynaecology Outpatient Ward of The University Hospital of Brasilia (Universidade de Brasília Hospital- HUB) for prenatal care routine ultrasound. They participated in the Music for Mothers Activities conducted by a multidisciplinary team composed of a Physician in charge of the ultrasound exam, a Musician and an Occupational Therapist. The data set was collected from the hospital records of this service. From these records were selected mothers whose patient profile included a minimum and maximum of 30 and 40 gestational weeks, mean gestational age was 34.7 weeks $( \pm 2.4)$ and foetuses showed no evidence of chromosomal, congenital or neurological abnormalities, or nutritional disorders. Not included were pregnant women whose foetuses showed signs of distress. This study was performed according to the Declaration of Helsinki (World Medical Association, 2008) and Resolution 510/2016 of the Brazilian National Research Council, and informed signed consent was obtained from all mothers.

\subsection{Outcome measures}

The record of movement and foetus HR was obtained by Doppler-ultrasound (Sonoline Versa Pro, Siemens, Germany). The examinations were performed by the same physician in a hospital facility, in the context of ultrasound monitoring of clinical care in pregnancy following the protocol of the maternal and child health program. The APIB scale was used to score the foetus' behavioural response by two behavioural dimensions that are likely to be observed by ultrasound: global movement (movement Factor-MOV) and attentional organization (organization Factor-ORG). MOV factor indicated the presence of gross motor response including limbs and trunk agitation. ORG factor indicated the presence of attentional response defined as body aligning, approach to or avoidance of the sound source.

\subsection{Procedures}


To establish the baseline of foetus behavior, each mother was instructed to be silent for at least 90 seconds and to remain at rest and in silence during the session until they were signalled to sing by the musician. The beginning of the presentation of all categories of sound stimuli started only after there was no record of foetus movement and stabilization of HR for at least 90 seconds [16]. The stimulus (song) had a duration that varied from 20 to 30 seconds, depending on the pace of the live playing music, with an interval between stages of 30 seconds. Each stage of the stimuli lasted a maximum of 60 seconds and a session had nearly five minutes duration.

The musical stimulus was a Brazilian folk lullaby in the public domain (the song "Se Essa Rua Fosse Minha", first strophe played and sung by a musician: 1) in slow tempo (Adagio) and 2) in fast tempo (Allegro); and 3) the pregnant women sang in slow tempo (Adagio). The sung music was accompanied by a four-stringed Brazilian musical instrument (a small guitar called a Cavaquinho), whose acoustic frequency spectrum varies between 295 and $3536 \mathrm{~Hz}$, a frequency spectrum similar to that of the female voice $[29,30]$. This arrangement using a musical instrument background stimulated a minimum of uniformity in tune and tempo for all the singers, so as to minimize the effect of gender of the voice and personal style of singing. The order in which stimuli were presented started with the slowest pace and increased gradually, not to stress the dyad and to prevent discomfort and agitation in both mother and foetus. The physician registered the HR values from the equipment data. The Occupational Therapist registered the foetus's behaviour and scored it according to the APIB scale after each trial.

The room's noise level was initially measured in silence during one ultrasound procedure with ambient noise level around $40 \mathrm{~dB}( \pm 20)$ (digital decibel meter, Lutron SL-4012). The external sound source, both the voice singing and the musical instrument, was at a distance of $100 \mathrm{~cm}$ from the mother's abdomen and was issued at an average intensity of $80 \mathrm{~dB}( \pm 20)$. Considering the level of noise in the womb may range from 70 to $90 \mathrm{~dB}$ and external noise (average intensity of both expected internal-womb and external noise), the external sound stimuli were issued at an intensity of noise level that would offset at least 10 to $40 \mathrm{~dB}$ of overall noise level [31].

\subsection{Statistical Methods}

Exploratory statistics were run in four stages: baseline plus the three stages of stimuli presentation. HR was treated as a continuous variable and "global movement" (MOV) and signs of "attention organization" (ORG) as dichotomous variables, registering the absence or presence of the foetal behavioural response. Spearman's rank-order correlation test correlated the APIB scores of the factors MOV and ORG with the rate of heartbeat and analysed the two experimental conditions (source and tempo) and baseline. The Friedman Test compared the four stages of the test by each Factor. The Wilcoxon Signed Ranks Test compared each Factor in pairs of stages. Two-way ANOVA was run, analysing all variables, with gestational age and basal HR as co-factors. Repeated measures ANOVA tested the time effect on the stages. All analyses were conducted using the IBM-SPSS package (Version 26.0).

\section{Results}

The three factors measured during the test, HR, ORG and MOV, showed a difference in patterns of response over all the four stages, basal and the three experimental conditions, as can be seen in Table 1 and Figure 1. The Friedman Test confirmed the overall significant changing profile among the stages with highly significant $\mathrm{p}$-values $(\leq .001)$ (Table 1$)$. A Spearman's rank-order correlation was run to determine the relationship between APIB Factors, HR and type of musical stimuli. There was a positive correlation between HR at baseline and at Stages 2 and 3, which was statistically significant $(\mathrm{rs}=.35, \mathrm{p}=.021$ and $\mathrm{rs}=.38, \mathrm{p}=.014$, respectively). MOV and HR correlated at Stage $1(\mathrm{rs}=.40, \mathrm{p}=.011)$. ORG response at Stages 1 and 2 were positively correlated $(\mathrm{rs}=.70, \mathrm{p}=.000)$. Two-Way ANOVA was run with all variables, having gestational age and basal HR as co-factors. In this analysis, gestational age had no effect upon the variables. Basal HR was only related to variations in HR of the external singer at Allegro ( $\mathrm{F}=2.165, \mathrm{p}=.050)$ and mother's Adagio at Stage $3(\mathrm{~F}=3.615, \mathrm{p}=0.003)$.

Table 1. Baseline and stages performance for the Factors HR (Mean \pm Standard Deviation) and APIB ORG and MOV (percentage based on presence of the foetus's responses). The stages are (1) external 
singer in slow pace (Adagio); (2) external singer in fast pace (Allegro); and (3) pregnant women singing in slow pace (Adagio). Friedman Test compared the four stages of the test by each Factor.

\begin{tabular}{lcccccc}
\hline \multicolumn{1}{c}{ Factors } & Baseline & $\begin{array}{c}\text { Stage 1 } \\
\text { Adagio }\end{array}$ & $\begin{array}{c}\text { Stage 2 } \\
\text { Allegro }\end{array}$ & $\begin{array}{c}\text { Stage 3 } \\
\text { Adagio } \\
\text { Maternal } \\
\text { voice }\end{array}$ & Chi-square & $p$ \\
\hline Heart Rate & $134.29 \pm 9.8$ & $139.07 \pm 10.9$ & $149.02 \pm 10.2$ & $130.88 \pm 7.8$ & 59.451 & .000 \\
APIB-Organization & $29.3 \%$ & $4.9 \%$ & $2.4 \%$ & $19.5 \%$ & 16.424 & .001 \\
APIB-Movement & $0 \%$ & $24.4 \%$ & $41.5 \%$ & $61 \%$ & 37.556 & .000 \\
\hline
\end{tabular}

Wilcoxon Signed Ranks Test compared each factor in pairs of stages (Table 2). The Factor ORG showed an increase from the beginning of the music presentation to the mother's Adagio and a pattern of habituation to the external singer. Meanwhile, the Factor MOV increased linearly with the session presentation schema, although it was significant just from baseline to first stage. No significant correlation was found between factors, but a marginal negative one between the variables HR and $\operatorname{MOV}(r=-.33, p=.051)$ at the mother's Adagio, indicating that there was an increase in MOV and a decrease in HR when the mother's voice started.

Table 2. Wilcoxon Signed Ranks Test comparing each Factor in pairs of stages, subtracting the latter from the former.

\begin{tabular}{clcc}
\hline Factor & & Z & $p$-Value \\
\hline \multirow{2}{*}{ Heart rate } & Stage1 minus Baseline & $-2.082^{\mathrm{a}}$ & .037 \\
& Stage 2 minus Stage 1 & $-5.021^{\mathrm{a}}$ & .000 \\
& Stage 3 minus Stage 2 & $-5.264^{\mathrm{b}}$ & .000 \\
\hline \multirow{3}{*}{ Organization } & Stage1 minus Baseline & $-2.887^{\mathrm{b}}$ & .004 \\
& Stage 2 minus Stage 1 & $-1.000^{\mathrm{b}}$ & .317 \\
& Stage 3 minus Stage 2 & $-2.333^{\mathrm{a}}$ & .020 \\
\hline \multirow{3}{*}{ Movement } & Stage1 minus Baseline & $-3.162^{\mathrm{a}}$ & .002 \\
& Stage 2 minus Stage 1 & $-1.807^{\mathrm{a}}$ & .071 \\
& Stage 3 minus Stage 2 & $-1.789^{\mathrm{a}}$ & .074 \\
\hline
\end{tabular}

Notes: a. Based on negative ranks; b. Based on positive ranks; c. Asymptotic Significance (2-tailed).

ANOVA repeated measure analysis, having time as factor and gestational age as covariate, found a statistically significant effect on foetuses' reaction to changing stimuli for all variables, $F_{(3,87)}$ $=12.29, \mathrm{p}=.000$. Mauchly's Test of Sphericity indicated that the assumption of sphericity had been violated for ORG, $\chi 2(2)=46.496, \mathrm{p}<.000$ and, therefore, a Greenhouse-Geisser correction was used. The tests of within-subjects effects showed a significant interaction effect of time and gestational age over all measures, $\mathrm{F}_{(22.84)}=2.040, \mathrm{p}=.003$. The estimated marginal means adjusted for the covariate gestational age and time effect over baseline plus three indicators are presented in Figure 1.

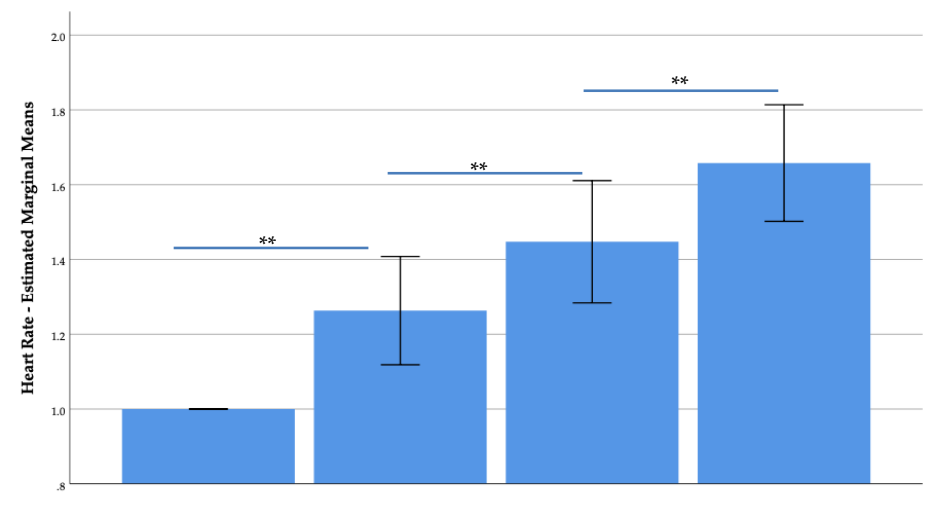




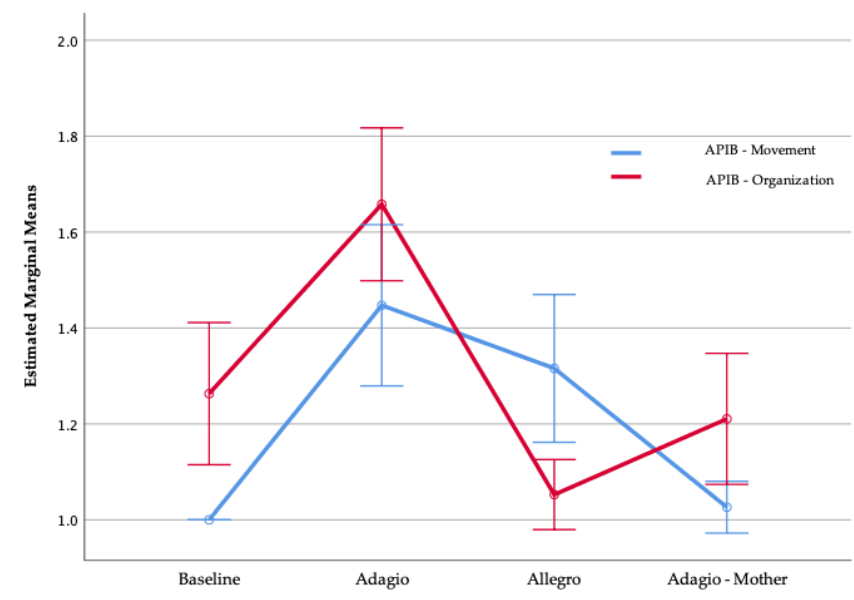

Figure 1. Estimated marginal means, adjusted for the covariate gestational age, of the performance of the three parameters Heart Rate variability (Top), APIB-Organization and APIB-Movement (Below) to four stages: baseline, external singer in slow tempo (Adagio); external singer in fast tempo (Allegro); and the pregnant women' voice in slow tempo singing to her foetus (Adagio-Mother). Error bar: 95\% Confidence Interval. Significant changes between stages are marked by ${ }^{*}(\mathrm{p}<.005)$ and ${ }^{* *}(\mathrm{p}<.000)$.

\section{Discussion}

Considering the nature of the HR and APIB measures, physiological and behavioural respectively, all showed a significant variation throughout the whole session; HR was the strongest parameter, thus corroborating previous studies [6, 18, 19]. The APIB scale's indicators proved sensitive to the foetus's reaction to the stimulation, as these responses match the pattern of premature infants presented [24, 25], especially for the Organization indicator, which discriminated responses among all stages. The MOV indicator was only significant for the change between baseline and the first presentation, with strong evidence of stimulus detection, similarly to what was found in other studies, suggesting a possible relationship with the maternal-foetus hormonal link [17, 32]. ORG was significantly different between the basal level and the first presentation (adagio external voice) and between the second and the third stage (Allegro external voice versus mother's Adagio), being possibly highly sensitive to source and to tempo.

The HR seems to keep pace with the tempo of the stimuli, accelerating as the external voice accelerated and decreasing to a lower level for the adagio mother's voice. This increment between baseline and second stage cannot be asserted, as it is sensitization, per se, to the continued stimulation or discrimination ability. This pattern was also present in previous studies [16, 32], although in this study it changed the pattern, with HR decreasing for maternal voice, suggesting a preference for this and thus also corroborating previous studies [14]. The increase in HR in Stage 2 may indicate that the foetuses were not yet habituated to the presence of music, and then the decrease in HR in Stage 3 indicated habituation, rather than any response to the differences in the stimuli between Stages 2 and 3. However, taking into account all three measures, among the habituation and/or sensitization patterns, the foetuses consistently showed changes from the baseline to the end of presentation, presenting more organized and active behaviour, with a low heart rate variability to the mothers' voice. Thus, an interpretative doubt remains about the return to basal levels in Stage 4 for HR, and about the increased movement and organization when the mothers sang the song, possibly signalling the foetuses' recognition skill and preference for the mothers' voice, or a decrease in the HR as the pace of the music also decreased.

The Two-Way ANOVA analyses considering basal HR and gestational age as factors showed that $\mathrm{HR}$ at baseline was positively and significantly related only to the external voice at allegro tempo and Stage 2 and mother's Adagio at Stage 3. This last result was expected, as the three factors showed different patterns, as stated above. However, it is noticeable that the basal level of HR did not have a significant effect upon the first stimulus presented in this analysis for all factors. Thus, the ORG factor 
was sensitive only between the first two and last two stages, possibly indicating a differential perception of sound source; therefore, the question remains of whether the foetuses were responding selectively to their mother or to external versus internal source. MOV was sensitive only to the change between the basal and first stage, although there was a general increment in MOV. However, the type of observed movements was unclear, and it was not possible to distinguish different categories of fine MOVs, such as hand to mouth, sucking, swallowing, etc. Gestational age showed no significant effect on the variables studied, indicating that the chosen range of gestational week was appropriate to this study. However, when another statistical model was tried, namely the ANOVA repeated measures, the estimated marginal means, adjusted for the covariate gestational age, showed an interaction between the time and gestational age. Furthermore, a within-subjects effect analysis revealed a general trend towards sensitization in HR, and MOV showed a habituation pattern, although ORG did not alter in relation to the weight of gestational age factor (Figure 1).

This study found different results from another study that investigated HR and stimulus orienting reflex in near-term foetuses using music (piano melodies with opposite contours), plus a natural Icelandic sentence and a chimera of the sentence with its spectral information replaced by broadband noise. All stimuli elicited HR deceleration in that study, but no clear difference was seen in response among the stimulus categories. The authors concluded that the piano melodies elicited a monophasic HR deceleration, indicating a stimulus orienting reflex, while the spoken sentence and its chimera evoked a sustained lower magnitude response, indicating a sustained attentional response or more focused information processing [33]. We can hypothesize that in our study we dealt with just with two dimensions, tempo and source, and this allowed a more focused analysis that found a clearer pattern of foetal response.

Among the limitations of this study, there were methodological issues concerning the use of APIB and Doppler-ultrasound for the study of the foetus's behavioural response, which must be considered. Firstly, the application of this scale while the foetus is inside the womb is limited to items based on global motor response to auditory stimulation, which may represent both auditory and attentional skills. Secondly, the observation of the foetus' response to auditory stimuli throughout the Doppler ultrasound is complicated, because the categorical analyses of the visual outputs must rely on a trained professional, limiting the operational definition and the application of inter-rater concordance tests. The accuracy of the motor response is also limited to the register of presence or absence of global movements, like startling movements or body dislocations that align and go back or forth from the sound source. Thirdly, it was possible to observe whether the foetuses were in general aligning, approaching or recoiling from the external sound source, but the limited number of subjects did not allow for compelling statistical analyses, and our choice was to keep the analysis limited to dichotomous variables. The fourth limitation is that the stimulus presentation order could not be counter-balanced, as the study design was planned not to create stress or agitation in mother and foetus, and thus the presentation order started with calm stimuli and gradually increased the pace not to stress the dyad. This study was run in a clinical setting, and it involved an emotionally delicate moment that highly anticipated by the pregnant women, given that they are going to make visual contact with their babies. As there were three different measures of the foetus's responses, we considered that a significant correlation among them with a reasonable theoretical justification would be adequate to support the study's conclusions.

Finally, the mothers' heart rate variability was not measured to allow comparison with their foetuses, and this must be addressed in future studies. Higher correlation has been found between mother-foetus HR variability when mothers were listening to auditory stimulation varying from .89 to .90 than in rest state .54 to .99 [2], although in another study a damping effect was observed in the foetal-maternal heart rate coupling in mothers who exercise regularly. This is most likely due to an increase in beat-to-beat differences, higher vagal tone and slower breathing rates in mother and foetus [3]. Research into foetal response to musical stimuli using HR synchronization as the only parameter must be interpreted with caution, and there is thus a need to have validated behavioural measures for use alongside physiological parameters. 


\section{Conclusion}

We conclude that in this study foetuses presented the ability to discriminate different sound sources and change in musical tempo by behavioural and physiological responses through sensitization and habituation patterns for both physiological and behavioural measures. Foetuses' attentional organization indicator detected and habituated to the external source stimulation and increased for maternal voice, showing a preference for the mother, in line with previous studies [13, 14], while global movement seemed not to respond to tempo, except at the beginning of the stimulation. There was no difference in response related to gestational age from 30 to 40 gestational weeks, but the interaction between time and gestational age changed the response profile for $\mathrm{HR}$, indicating that preterm and near-term foetuses present a different pattern of response to musical stimuli. This is suggestive for future studies, which might consider analysing each gestational week separately.

This paper replicates former studies, but innovates in applying a neonatal behavioural scale, the APIB, hitherto not yet applied in this context [19]. This is important, as this research field is searching for new ways to understand the meaning of foetuses' reactions inside the womb through Doppler [34]. We consider that more interactive studies with foetuses should be carried out to refine the definition of gross and fine motor categories of response, since ultrasound tests are improving in precision. The APIB factors of movement and organization presented were suitable as a measuring tool to study the foetal behavior in the last 10 weeks before delivery. Application of the scale can be implemented as a parameter for research in auditory perception in foetuses, and follow-up after delivery can take place using the same instrument. Moreover, music stimulation has become a wellsupported resource for improvement of premature infants' and foetuses' neurodevelopment, considering that it might promote neurobiological processes such as modulation of synaptic plasticity and might facilitate the differentiation, activation, readjustment and growth of neurons; therefore, its outcomes should be investigated [35-37].

Author Contributions: Conceptualization, M.L.L. and R.D.F.; Methodology, M.L.L. and R.D.F; Formal Analysis, R.M.T., J.A.L.J., M.L.L. and R.D.F; Investigation, M.L.L. and R.D.F; Resources, M.L.L. and R.D.F; WritingOriginal Draft Preparation, R.M.T.; Writing-Review \& Editing, R.M.T., J.A.L.J., M.L.L. and R.D.F; Project Administration, M.L.L..

Funding: This research was supported by a grant from Fundação de Apoio a Pesquisa do Distrito Federal/Conselho Nacional de Pesquisa, linked to the Ministry of Science, Technology and Innovation of Brazil (grant number 193.000.326/2009) awarded to Rosana Maria Tristão.

Acknowledgments: We would like to thank Luis Gustavo Rosas de Melo, MD, for his engagement and involvement in helping with the ultrasound exams, and we are grateful to all the parents that gave their consent and participated in this study.

Conflicts of Interest: The authors declare no conflict of interest.

Abbreviations

HR Heart Rate

APIB Assessment of Preterm Infants Behavior

ORG Attentional Organization

MOV Movement

\section{References}

[1] Kisilevsky BS, Low JA. Human Fetal Behavior: 100 Years of Study. Dev Rev 1998; 18: 1-29.

[2] Kisilevsky BS, Brown CA. Comparison of fetal and maternal heart rate measures using electrocardiographic and cardiotocographic methods. Infant Behav Dev 2016; 42: 142-151. 
[3] Van Leeuwen P, Gustafson KM, Cysarz D, et al. Aerobic Exercise during Pregnancy and Presence of Fetal-Maternal Heart Rate Synchronization. PLoS ONE 2014; 9: e106036.

[4] Mishra S, Roy TS, Wadhwa S. Morphological and morphometrical maturation of ventral cochlear nucleus in human foetus. J Chem Neuroanat 2018; 93: 38-47.

[5] Kisilevsky BS, Pang L, Hains SMJ. Maturation of human fetal responses to airborne sound in low- and high-risk fetuses. Early Hum Dev 2000; 58: 179-195.

[6] Querleu D, Renard X, Versyp F, et al. Fetal hearing. Eur J Obstet Gynecol Reprod Biol 1988; 28: 191-212.

[7] Zimmer EZ, Fifer WP, Kim YI, et al. Response of the premature fetus to stimulation by speech sounds. Early Hum Dev 1993; 33: 207-215.

[8] Groome LJ, Mooney DM, Holland SB, et al. Behavioral state affects heart rate response to low-intensity sound in human fetuses. Early Hum Dev 1999; 54: 39-54.

[9] Lecanuet JP, Granier-Deferre C, DeCasper AJ, et al. [Fetal perception and discrimination of speech stimuli; demonstration by cardiac reactivity; preliminary results]. C R Acad Sci III 1987; 305: 161-164.

[10] Lecanuet J-P, Granier-Deferre C, Jacquet A-Y, et al. Prenatal discrimination of a male and a female voice uttering the same sentence. Early Dev Parent 1993; 2: 217-228.

[11] Lecanuet J-P (ed). Fetal development: a psychobiological perspective. Hillsdale, N.J: L. Erlbaum Associates, 1995.

[12] Lecanuet J-P, Schaal B. Fetal sensory competencies. Eur J Obstet Gynecol Reprod Biol 1996; 68: $1-23$.

[13] DeCasper A, Fifer W. Of human bonding: newborns prefer their mothers' voices. Science 1980; 208: 1174-1176.

[14] DeCasper AJ, Spence MJ. Prenatal maternal speech influences newborns' perception of speech sounds. Infant Behav Dev 1986; 9: 133-150.

[15] Moon C, Cooper RP, Fifer WP. Two-day-olds prefer their native language. Infant Behav Dev 1993; 16: 495-500.

[16] Sontag LW, Steele WG, Lewis M. The fetal and maternal cardiac response to environmental stress. Hum Dev 1969; 12: 1-9.

[17] Gebuza G, Zaleska M, Kaźmierczak M, et al. The effect of music on the cardiac activity of a fetus in a cardiotocographic examination. Adv Clin Exp Med 2018; 27: 615-621.

[18] Lai J, Nowlan NC, Vaidyanathan R, et al. Fetal movements as a predictor of health. Acta Obstet Gynecol Scand 2016; 95: 968-975.

[19] Fernando KL, Mathews VJ, Varner MW, et al. Robust estimation of fetal heart rate variability using doppler ultrasound. IEEE Trans Biomed Eng 2003; 50: 950-957.

[20] Antonets VA, Kazakov VV. On noninvasive assessment of acoustic fields acting on the fetus. Acoust Phys 2014; 60: 342-347.

[21] Parncutt R. Mother-infant attachment, musical idol worship, and the origins of human behaviour. Music Sci 2018; 22: 474-493.

[22] Gebuza G, Dombrowska A, Kaźmierczak M, et al. The effect of music therapy on the cardiac activity parameters of a fetus in a cardiotocographic examination. J Matern Fetal Neonatal Med 2017; 30: 2440-2445. 
[23] López-Teijón M, García-Faura Á, Prats-Galino A. Fetal facial expression in response to intravaginal music emission. Ultrasound 2015; 23: 216-223.

[24] Als H. A Synactive Model of Neonatal Behavioral Organization:: Framework for the Assessment of Neurobehavioral Development in the Premature Infant and for Support of Infants and Parents in the Neonatal Intensive Care Environment. Phys Occup Ther Pediatr 1986; 6: 3-53.

[25] Als H, Butler S, Kosta S, et al. The Assessment of Preterm Infants' Behavior (APIB): Furthering the understanding and measurement of neurodevelopmental competence in preterm and full-term infants. Ment Retard Dev Disabil Res Rev 2005; 11: 94-102.

[26] Brazelton TB, Nugent JK. Neonatal Behavioral Assessment Scale (Clinics in Developmental Medicine). Mac Keith Press.,

http://search.ebscohost.com/login.aspx?direct=true\&scope=site\&db=nlebk\&db=nlabk\&AN=5 03755 (accessed 3 June 2020).

[27] Costa R, Figueiredo B, Tendais I, et al. Brazelton Neonatal Behavioral Assessment Scale: A psychometric study in a Portuguese sample. Infant Behav Dev 2010; 33: 510-517.

[28] Cardoso SMS, Kozlowski L de C, de Lacerda ABM, et al. Newborn physiological responses to noise in the neonatal unit. Braz J Otorhinolaryngol 2015; 81: 583-588.

[29] Richards TA. The Cavaquinho Chord Bible: DGBD Standard Tuning 1,728 Chords. Cabot Books, United Kingdom: 2008 ISBN 978-1-906207-09-0

[30] Pabon P, Ternström S. Feature Maps of the Acoustic Spectrum of the Voice. J Voice 2020; 34: 161.e1-161.e26.

[31] Parga JJ, Daland R, Kesavan K, et al. A description of externally recorded womb sounds in human subjects during gestation. PLOS ONE 2018; 13: e0197045.

[32] Zimmer EZ, Divon MY, Vilensky A, et al. Maternal exposure to music and fetal activity. Eur J Obstet Gynecol Reprod Biol 1982; 13: 209-213.

[33] Granier-Deferre C, Ribeiro A, Jacquet A-Y, et al. Near-term fetuses process temporal features of speech: Fetuses process temporal features. Dev Sci 2011; 14: 336-352.

[34] Department of Obstetrics and Gynecology, Clinical Hospital "Sveti Duh", Medical School University of Zagreb, Zagreb, Croatia, Kurjak A, Stanojevic M, et al. Is four-dimensional (4D) ultrasound entering a new field of fetal psychiatry? Psychiatr Danub 2019; 31: 133-140.

[35] Sacks O. Musicophilia: tales of music and the brain. Rev. and expanded, 1st Vintage Books ed. New York: Vintage Books, 2008.

[36] Rickard NS, Toukhsati SR, Field SE. The Effect of Music on Cognitive Performance: Insight From Neurobiological and Animal Studies. Behav Cogn Neurosci Rev 2005; 4: 235-261.

[37] Haslbeck FB, Karen T, Loewy J, et al. Musical and vocal interventions to improve neurodevelopmental outcomes for preterm infants. Cochrane Database Syst Rev. Epub ahead of print 14 November 2019. DOI: 10.1002/14651858.CD013472. 